\title{
i Engaging as an innovative approach to engage patients in their own fall prevention care
}

\author{
Huey-Ming Tzeng' \\ Chang-Yi Yin ${ }^{2}$ \\ 'College of Nursing, Washington \\ State University, Spokane, WA, USA; \\ ${ }^{2}$ Department of History, Chinese \\ Culture University, Taipei, Taiwan
}

Correspondence: Huey-Ming Tzeng College of Nursing, Washington State University, PO Box 1495, Room I67, Spokane, WA 99210-1495, USA

Tel +l 7343580358

Email tzenghm@gmail.com

\author{
This article was published in the following Dove Press journal: \\ Patient Preference and Adherence \\ 13 May 2014 \\ Number of times this article has been viewed
}

\begin{abstract}
Decreasing patient fall injuries during hospitalization continues to be a challenge at the bedside. Empowering patients to become active participants in their own fall prevention care could be a solution. In a previous study, elderly patients recently discharged from a United States hospital expressed a need for nurses to give and repeat directives about fall prevention; when the nurse left a brochure on the topic, but did not provide any (or limited) verbal explanations about the content or the importance of the information, the patient felt that the information was insufficient. To address patients' needs, we developed " $i$ Engaging", a Web-based software application for use at the bedside. $i$ Engaging is an innovative approach that is used to engage patients in their own fall prevention care during hospital stays. The application was designed based on the assumption that patients are the best and most critical sources of information about their health status. $i$ Engaging has not yet been tested in clinical trials.
\end{abstract}

Keywords: patient, safety, hospital, nursing care, fall, consumer involvement

\section{Introduction}

Patients 65 years and older who were recently discharged from a United States hospital expressed a need for nurses to give and repeat directives about fall prevention. ${ }^{1}$ These patients found that when the nurse left a brochure on the topic, but did not provide any (or limited) verbal explanations about the content or importance of the information, the patient felt that the information was insufficient. ${ }^{1}$ Patients are entitled to receive quality fall prevention care tailored to their risk for falling, and such patient centeredness could lead to safe hospital stays. ${ }^{2}$ Patient engagement in their health care could translate into measurable improvements in safety and quality. Thus, there are shared responsibilities and accountabilities between each patient and his or her clinicians that make active partnerships effective in care delivery. ${ }^{3-5}$ An example of promoting patient engagement is the use of interactive technology or software that allows patients to rehearse attending an outpatient clinician visit. ${ }^{5}$ Regardless of their racial, economic, or educational background, patients who have low levels of motivation to engage in their own care (for example, in postdischarge care) may be at increased risk of being rehospitalized within 30 days of discharge. ${ }^{6}$

Robinson et $\mathrm{al}^{7}$ have highlighted a potential gap in clinical practice in which encounters between patients and clinicians should be (but have not been) a setting for new information exchange to promote patient empowerment and shared decision making. Clinical encounters via a Web-based program could be a venue for such exchanges, which might lead to better care outcomes (for example, to fewer inpatient injurious falls). To address patients' need for this type of information, we collaborated to invent an 
innovative approach that may lead to fewer inpatient falls and fall-related injuries.

The purpose of this paper is to describe the design of the " $i$ Engaging" prototype. As a Web-based software application for use at the bedside, $i$ Engaging is meant to engage patients in their own fall prevention care during hospital stays. In other words, it is an interactive digital approach that is used to promote patient empowerment and shared decision making. $i$ Engaging is being developed with the ultimate goal of reducing the risk of falls among individuals aged 65 years and older, and it holds relevance in inpatient and community-based settings and across varied languages and cultures if user testing shows promise.

\section{The significance of fall-related issues among hospitalized older adults}

In 2010 in the US, $31.7 \%$ of adults aged 65 years or older fell and experienced injury within the past year. ${ }^{8}$ Inpatient injurious falls that occur in US acute care hospitals are the most prevalent in-hospital adverse event, with rates as high as 21.4 falls per 1,000 patient days for older inpatients. ${ }^{7-14}$ Inpatients are at a greater risk of falling than those in the general population, mainly due to acute illness and being unfamiliar with the hospital environment. ${ }^{15-17}$ Falls arise from multiple factors that are relevant to the environment of care and to patients' individual characteristics (for example, impaired cognition, mobility, gait, and balance). ${ }^{18}$ A recent study ${ }^{19}$ conducted in a US acute care hospital showed that $92 \%$ of all inpatient falls could be prevented; about $43 \%$ of all inpatient falls are accidental falls related to environmental factors, $49 \%$ are anticipated physiological falls related to intrinsic factors, and only $8 \%$ are unanticipated physiological or intentional falls.

Multifactorial interventions to prevent inpatient falls in hospital settings may decrease injurious fall incidents; however, interventions that are a key for success in preventing these falls have not yet been specified. ${ }^{15,20-25}$ Recent studies $^{26-28}$ suggested that patient engagement could lead to better health outcomes. However, the effectiveness of approaches that engage patients in fall prevention care for reducing fall incidents during hospital stays has not yet been tested empirically in acute care settings.

\section{Using tablet computers to engage patients in bedside care}

Many hospitalized patients have significant idle/inactive time between health care provider visits, tests, treatments, visits with family or friends, and rest. The inactive time could be used for patients to engage in their care by accessing health education, tailored patient information (for example, information regarding a patient's medications), and the patient's personal health record (PHR) to manage medications and postdischarge appointments via electronic bedside communication devices such as tablet computers. ${ }^{29-32}$ Previous studies ${ }^{11,33,34}$ suggested that technology (for example, digital video education materials) can help deliver patient-centered fall prevention care, including to older adults; however, these studies did not use tablet computers to engage patients.

Greysen et $\mathrm{al}^{29}$ conducted a pilot project to explore inpatient satisfaction levels with bedside tablets (Apple iPad 2; Apple Inc., Cupertino, CA, USA) and barriers to usability. They also evaluated use of tablets to deliver PHR access (Epic MyChart; Epic Systems Corporation, Verona, WI, USA) and online inpatient education modules about hospital safety (developed by Emmi Solutions LLC, Chicago, IL, USA). This study enrolled a total of 30 patients; only eight $(28 \%)$ were aged 60 years or older. A total of $17(60 \%)$ were women, six (22\%) owned tablet computers, and 26 (87\%) owned any mobile device (tablet, laptop, or Smartphone). Twenty-six (87\%) required 30 minutes or less for basic tablet orientation, and $70 \%$ required 15 minutes or less. Participants aged 50 years or older $(n=12 ; 42 \%)$ were less likely to complete the orientation in 15 minutes $(25 \%)$ compared with those under 50 years old (79\%). Age was not found to be a significant factor in using tablet interventions to complete at least one PHR function or to complete health education modules. Twenty-seven participants $(90 \%)$ reported high overall levels of satisfaction with the tablet computer device. Greysen et $\mathrm{al}^{29}$ concluded that tablet computers could be used in inpatient care settings for clinically meaningful tasks (for example, in discharge planning and inpatient education) by patients without hardware issues.

\section{$i$ Engaging}

$i$ Engaging is a Web-based software application for use at the bedside to engage patients in taking an active role in fall prevention during hospitalization (Figure 1). This application contains auditory content, and a functional prototype was designed for patients who read and speak English. For demonstration purposes, a short video clip (80 seconds in length) was created (https://www.youtube.com/watch? $\underline{v}=2 \mathrm{zvqNBDkxlY} \&$ feature=youtube gdata player). This application works well on any kind of personal computer or Apple computer device (Apple, Inc., Cupertino, CA, USA) (desktop or laptop computers and Android tablets [Google Inc., Mountain View, CA, USA]). However, the 
A

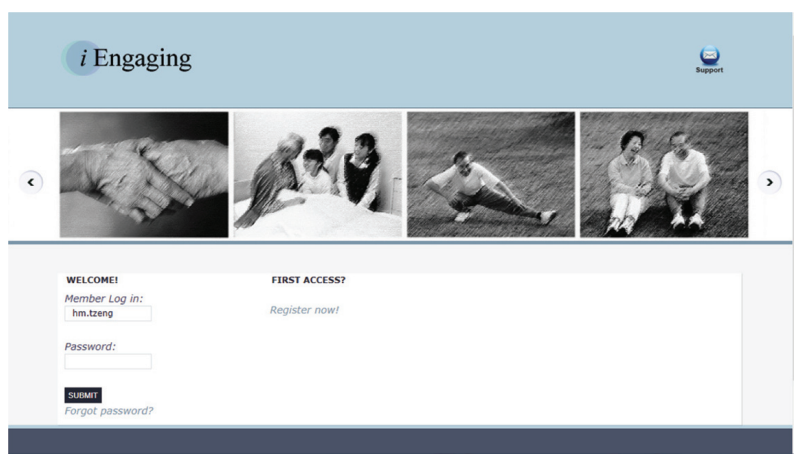

C

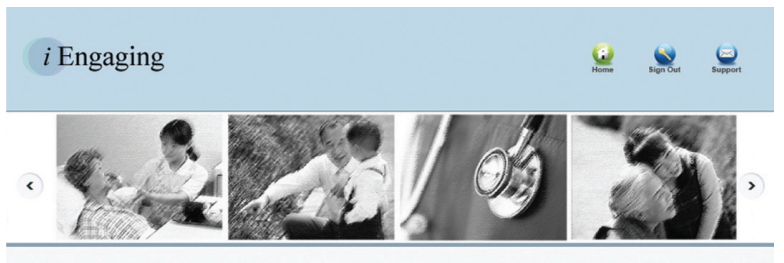

Welcome hm tzeng, what would you like to do?

- Review my previously conducted assessments

Review my previously established fall prevention plans

2 Continue my unfinished assessment

- I am ready to take my assessment

E

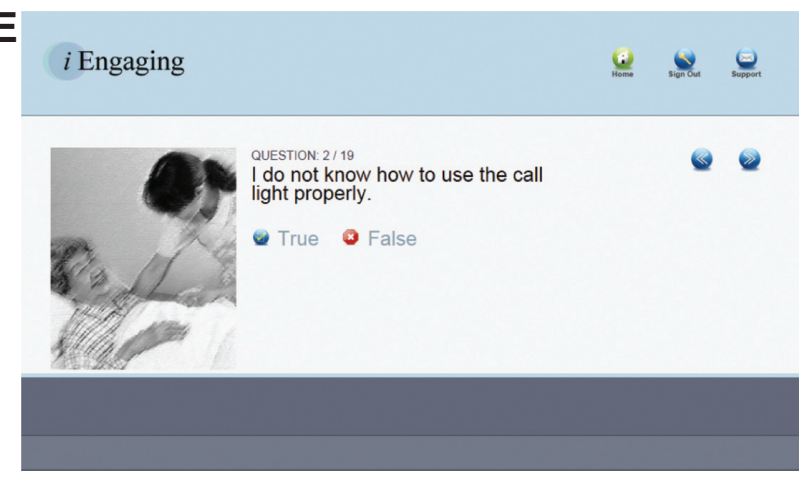

G

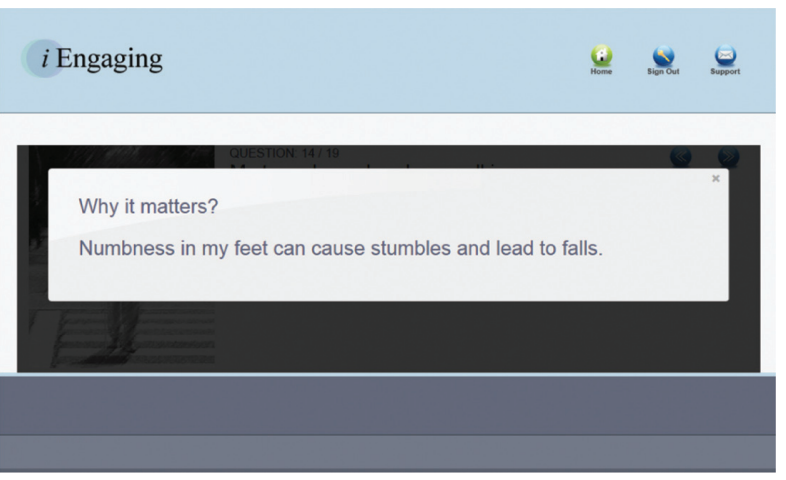

Figure I (Continued)
B

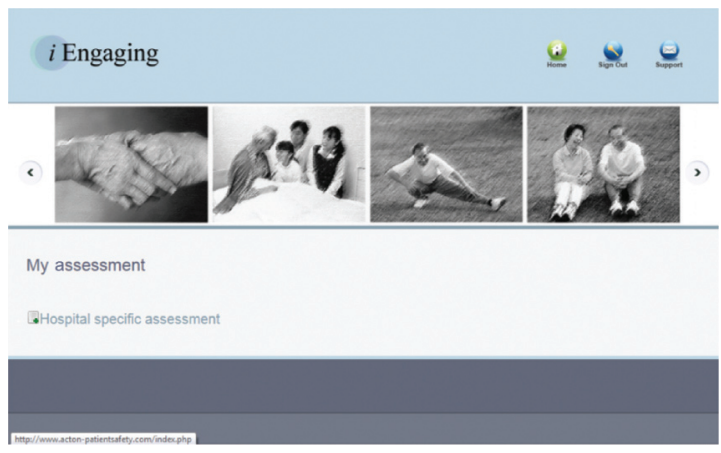

D

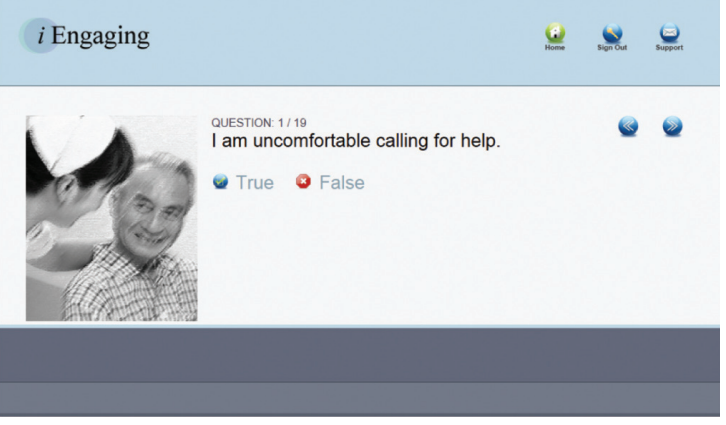

$\mathbf{F}$

$i$ Engaging

(4)

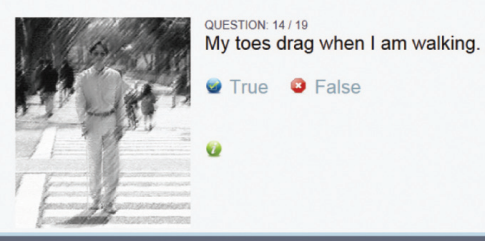

H

$i$ Engaging

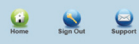

Verify the risks I have identified

\begin{tabular}{lc}
\hline My fall risk factors & My response \\
\hline I am uncomfortable calling for help. & TRUE \\
\hline I do not know how to use the call light properly. & TRUE \\
\hline I have fallen time(s) in the past 7 days. & 1 \\
\hline I am concerned about falling. & TRUE \\
I have problems with heart rate or rhythm. & TRUE \\
\hline I am sometimes forgetful. & TRUE \\
\hline I sometimes have to rush to the bathroom. & TRUE \\
I often feel sad. & TRUE \\
\hline I nfton hava Nizrinoce whan I ctand un & TRIII
\end{tabular}




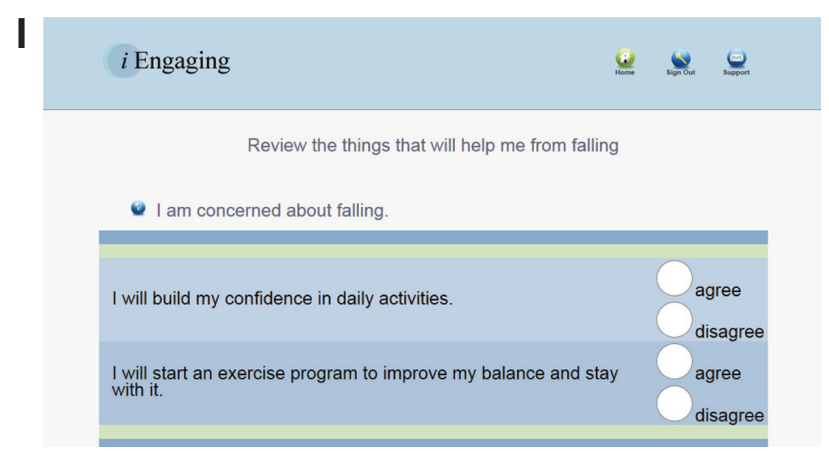

Figure I $i$ Engaging screenshots.

automatic auditory content of this application does not work on the iPad and similar Apple devices (Apple, Inc.). It is designed to help patients understand the causes of falling and to make decisions to reduce their risks. $i$ Engaging addresses the needs of patients aged 65 years or older, given their high nonfatal fall injury risk. ${ }^{35}$

The main features of this interactive application include: 1) it provides an inpatient fall risk assessment (Table 1);2) it identifies a set of things selected by patients that they can do themselves to prevent falls for each identified risk factor; and 3) it has the capability of printing an individualized fall prevention plan. A sample of an individualized fall prevention plan is shown in Figure 2. For illustration purposes, this plan includes all fall risk factors and all the things patients may do to prevent falling.

The fall risk assessments and things patients can do to address their identified fall risks (Table 1 and Figure 2)

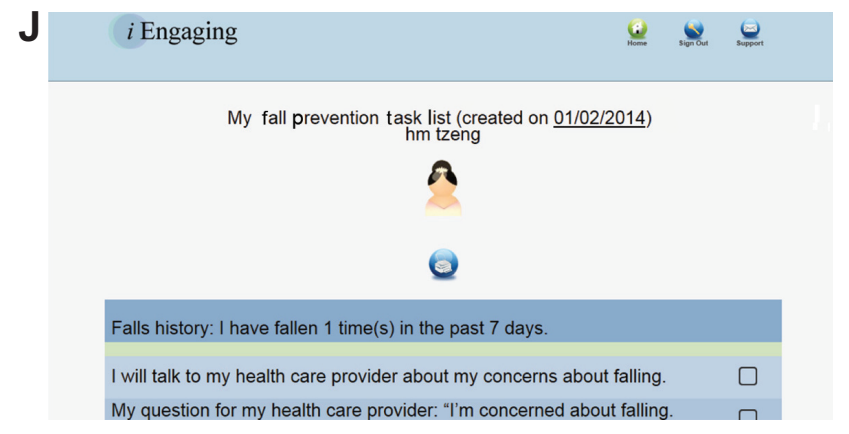

were developed based on the findings and principles from previous studies. ${ }^{15,16,36-43}$ The first version of the patient fall risk assessment was primarily developed from tools created by Rubenstein et $\mathrm{al}^{41}$ and by the Spokane Regional Health District, Fall Free Spokane Coalition..$^{42}$ The patient fall risk assessment and corresponding preventive approaches are designed in such a way to make them relevant for patients during hospital stays. From mid-2013 to early 2014, the authors have informally solicited feedback from more than ten patient partners aged 65 years or older at a senior and community center located in eastern Washington, USA and 20 clinician, hospital, or nurse administrator partners from three hospitals located in eastern Washington, USA. Clinician, hospital, and nurse administrator partners, as content experts, also reviewed the patient fall risk assessment and corresponding preventive approaches for their accuracy and relevancy. As a result, some wording and layout

Table I $i$ Engaging fall risk self-assessment questions

\begin{tabular}{|c|c|}
\hline Category & Risk factor question (responding to each statement by indicating "true" or "false") \\
\hline \multirow[t]{2}{*}{ Call light use problems } & I am uncomfortable calling for help. \\
\hline & I do not know how to use call light properly. \\
\hline \multirow[t]{2}{*}{ Fall history } & I have fallen __ times in the past 7 days. (True $=$ I or more times). \\
\hline & I am concerned about falling. \\
\hline Heart problems & I have problems with heart rate or rhythm. \\
\hline Memory problems & I am sometimes forgetful. \\
\hline Incontinence & I sometimes have to rush to the bathroom. \\
\hline Depression & I often feel sad. \\
\hline Blood pressure problems & I often have dizziness when I stand up. \\
\hline \multirow[t]{3}{*}{ Mobility problems } & I feel unsteady when I am walking. \\
\hline & I need to steady myself by holding onto furniture or walls when walking. \\
\hline & I do not use my cane or walker to get around safely. \\
\hline \multirow[t]{2}{*}{ Foot problems } & I have lost some feeling in my feet. \\
\hline & My toes drag when I am walking. \\
\hline \multirow[t]{3}{*}{ Medication problems } & Currently, I take four or more prescriptions or over-the-counter medications daily. \\
\hline & I take medicine that sometimes makes me feel lightheaded or more tired than usual. \\
\hline & I take medicine to help me sleep or improve my mood. \\
\hline Vision problems & I have vision problems not corrected by glasses or contact lenses. \\
\hline Hearing problems & I have hearing problems not corrected by hearing aids. \\
\hline
\end{tabular}


A

$i$ Engaging

My fall prevention task list (created on 04/29/2014)
Hm tzeng

a

Q

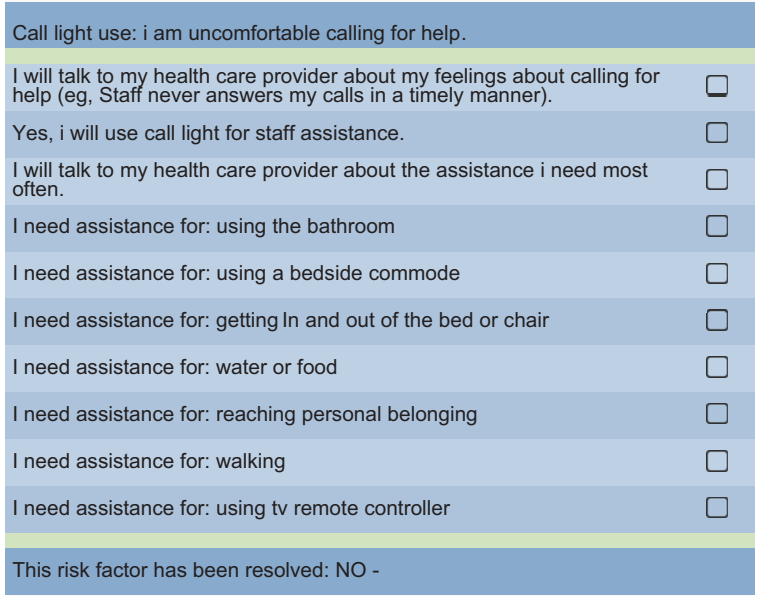

Call light use: i do not know how to use the call light properly.

C

I will talk to my health care provider about my memory problem.

My question for my health care provider: "sometimes, i am forgetful. Should i be screened for this?"

This risk factor has been resolved: NO -

Incontinence: $\mathrm{i}$ sometimes have to rush to the bathroom.
I will talk to my health care provider about my risk of falling due to this-
rushing to the bathroom.
This risk factor has been resolved: NO -

Depression: i often feel sad.

I will talk to my health care provider about my sadness.

$\sqsubset$

This risk factor has been resolved: NO -

\section{Blood pressure: $i$ often have dizziness when i stand up.}

Talk to my health care provider about my symptoms.

I will try sleeping with extra pillows to raise my head. I will talk to my health care provider whether i need to limit my liquid
intake. Drink $6-8$ glasses of water or low-calorie drinks each day. intake. Drink 6-8 glasses of water or low-calorie drinks each day.
my question for my health care provider: "i read that some medicines can
make me dizzy Do you think any of mine might be a problem?"

I will get out of bed slowly. First sit up, then sit on the side of the bed and I will get out of bed slowly. First sit up, th.
march legs up and down, then stand up.

I will take my time when changing position, such as when getting up from a chair.

I will sit down when washing, showering, dressing, or working in the kitchen.

I will exercise gently before getting up (move my feet up and down and clench and unclench my hands) or after standing (march in place).

\section{B} I will talk to my health care provider about my need to learn how to use $\quad \square$ call light.

I must demonstrate how to use call light properly.

This risk factor has been resolved: no -

Falls history: i had fallen at least once in the past 7 days
I will talk to my health care provider about my concerns about falling.
My question for my health care provider: "'m concerned about falling.
What are things i can do to keep myself from falling?"
My question for my health care provider: "iknow a fall can be serious.
I Want to take a fall prevention class. What do you recommend?"
This risk factor has been resolved: no -

Falls history: i am concerned about falling.
I will build my confidence in daily activities.
I will start an exercise program to improve my balance and stay with it.
This risk factor has been resolved: NO -

\section{Heart problem: $i$ have problems with heart rate or rhythm.}

I will talk to my health care provider about the types and amount of Exercise that are right for me.

This risk factor has been resolved: NO -

Memory: i am sometimes forgetful.

D

I will make sure i have something to hold onto when i stand up.

I will not walk if $\mathrm{i}$ feel dizzy.

I will avoid taking very hot baths or showers.

ᄃ

IWil avoid taking vary hot baths or showers.

This risk factor has been resolved: NO -

Mobility: i feel unsteady when i am walking.
I will talk to my health care provider about my need to see physical
therapist.
I will start an exercise program to improve my stability and balance.
This risk factor has been resolved: NO -

Mobility: i need to steady myself by holding onto furniture or walls when walking. I will talk to my health care provider about my need to see physical therapist.

I will start an exercise program to improve my stability and balance.

$\sqsubset$

This risk factor has been resolved: NO -

Mobility: i do not use my cane or walker to get around safely.

I will talk to my health care provider about my need to see physical therapist.

Figure 2 (Continued) 
E

I will talk to my health care provider about my foot problem.
I will wear nonslip footwear.
This risk factor has been resolved: NO -

Foot problem: my toes drag when i am walking.
I will talk to my health care provider about my foot problem.
This risk factor has been resolved: NO -

Medication: currently, i take four or more prescriptions or over-the-counter
medications daily.
I will have my health care provider look at all the medicines i take, even
over-the-counter medicines.
I will have a full review of medications every year with a pharmacist
or physician or with any medication changes.
This risk factor has been resolved: NO -

Medication: i take medicine that sometimes makes me feel light-headed or more
tired than usual.
I will learn which of my medications can cause drowsiness, dizziness, or
weakness as a side effect.
I will talk to my health care provider about my medicines that can make
me dizzy.
My question for my health care provider: "i read that some medicines can
make me dizzy. Do you think any of mine might be a problem?"
This risk factor has been resolved: NO -

Medication: i take medicine to help me sleep or improve my mood.

Figure 2 i Engaging individualized fall prevention plan.

changes were made to increase the patient-centeredness and ease of use for patients 65 years or older, including those with little or no experience in using computers and tablets.

$i$ Engaging is designed to have a health care provider help the patient set up an $i$ Engaging account (this takes about 5 minutes), which the patient will use to complete a risk assessment profile and generate a printable individualized fall prevention plan (about 5 minutes). Patients may continue to use $i$ Engaging as they like and as their conditions change. The preferred platform will be a touch-screen, easy-to-clean tablet computer. The application and information will be accessible to patients via mobile devices (tablet, laptop, or Smartphone) since the application uses a browser-based approach. Clinical trials on the effectiveness of $i$ Engaging in fall prevention are warranted and will provide assistance to patients while using the tool.

\section{Conclusion}

$i$ Engaging is designed based on the assumption that patients are the best and most critical source of information regarding

\section{$\mathbf{F}$}

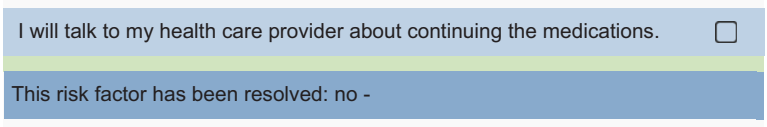

Vision: i have vision problems not corrected by glasses or contact lenses.

I will schedule an eye exam. It is recommended to schedule an eye exam at least every 2 years or with any vision change.

This risk factor has been resolved: no -

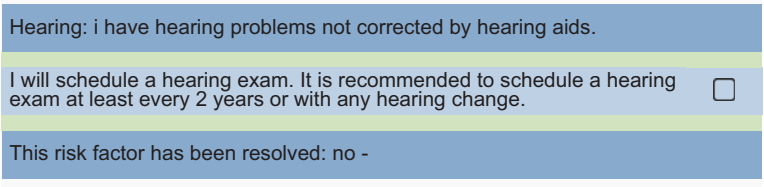

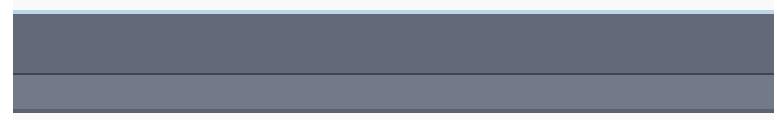

their health status, and they have the right to make their own decisions about their own care. ${ }^{4}$ As part of the iterative development process, future developments will include improved fidelity for the digital reader (auditory component), and incorporating a game-like approach or using storylines and illustrations that demonstrate examples in which unaddressed fall risks lead to bad outcomes (for example, broken legs or upper arms that are due to falls related to uncorrected vision problems). The team is refining the registration page using a dramatic hook to increase the user's motivation to register with the application; this update is important to ensure that users recognize the importance of the content they receive when they register at the Website. A new application name or a subtitle on the registration page specifically linked to fall prevention is warranted to increase digital search efficiency.

In order to serve as a linguistically appropriate patient education tool for fall prevention, the content of $i$ Engaging may be translated into other languages after the effectiveness of its English version in preventing falls during hospital stays is demonstrated. Hospital inpatient units often adopt 
a multifocal patient education approach to reduce patient fall risks using posters, handouts, whiteboard or chalkboard reminders, personal or group dialogue, and regular reminders during hourly room checks. ${ }^{15} i$ Engaging could be a natural fit as a component of the fall risk reduction protocol or initiatives underway in hospital settings. The design of $i$ Engaging is continuously being refined based on the findings of formal and informal user surveys for future clinical trials when funding is available. This Web-based software application may be tailored to be relevant in both inpatient and community-based settings and to serve as a continuum care tool for fall prevention.

\section{Disclosure}

The authors are not planning to make the idea/invention commercially available, and they intend to publicly disclose the invention via this paper. The first author owns the trademark of $i$ Engaging (serial number: 85962879; approved by the United States Patent and Trademark Office [USPTO]). Washington State University, Pullman, WA, USA filed a provisional application to the USPTO on July 22, 2013, titled " $i$ Engaging - A patient engagement application for patient fall prevention training". Both authors are the inventors and own the copyright of the content of this invention. Both authors do not intend to commercialize this invention and have no financial gains from this invention at this time. $i$ Engaging is a free Webbased application. The authors report no other conflicts of interest in this work.

\section{References}

1. Tzeng HM, Yin CY. Perspectives of recently discharged patients on hospital fall-prevention programs. J Nurs Care Qual. 2009;24(1):42-49.

2. Tzeng HM, Yin CY. Patient engagement in hospital fall prevention. Nurs Econ. 2014. In press.

3. Agency for Healthcare Research and Quality [webpage on the Internet]. Guide to patient and family engagement in hospital quality and safety. Rockville, MD: Agency for Healthcare Research and Quality; 2013. Available from: http://www.ahrq. gov/professionals/systems/hospital/engagingfamilies/index.html?utm medium=email\&utm_source=govdelivery. Accessed February 18, 2014.

4. Nursing Alliance for Quality Care. The Nursing Alliance for Quality Care National Consensus Conference, Herndon, VA, 12-13 November 2012. Silver Spring, MD: American Nurses Association; 2012. Available from: http://www.naqc.org/Main/Resources/2012-NursesContributionsFosteringSuccessfulPatientEngagement.pdf. Accessed February 2, 2014.

5. Carlson D, Patterson L, Holm M. The new rules of engagement. Washington Nursing Commission News. 2014;8(1):14.

6. Stempniak M. Predictive modeling: Patient activation could be a big step forward in reducing rehospitalizations. Hosp Health Netw. 2014;88(1):21.

7. Robinson L, Newton JL, Jones D, Dawson P. Self-management and adherence with exercise-based falls prevention programmes: a qualitative study to explore the views and experiences of older people and physiotherapists. Disabil Rehabil. 2014;36(5):379-386.
8. Centers for Disease Control and Prevention. The State of Aging and Health in America 2013. Atlanta, GA: Centers for Disease Control and Prevention, US Dept of Health and Human Services; 2013. Available from: http://www.cdc.gov/features/agingandhealth/state_of_aging and_health_in_america_2013.pdf. Accessed March 8, 2014.

9. Haines TP, Hill KD, Bennell KL, Osborne RH. Additional exercise for older subacute hospital inpatients to prevent falls: benefits and barriers to implementation and evaluation. Clin Rehabil. 2007;21(8): $742-753$.

10. Halfon P, Eggli Y, Van Melle G, Vagnair A. Risk of falls for hospitalized patients: a predictive model based on routinely available data. J Clin Epidemiol. 2001;54(12):1258-1266.

11. Hill AM, McPhail S, Hoffmann T, et al. A randomized trial comparing digital video disc with written delivery of falls prevention education for older patients in hospital. J Am Geriatr Soc. 2009;57(8): $1458-1463$.

12. Milisen K, Staelens N, Schwendimann R, et al. Fall prediction in inpatients by bedside nurses using the St. Thomas's Risk Assessment Tool in Falling Elderly Inpatients (STRATIFY) instrument: a multicenter study. J Am Geriatr Soc. 2007;55(5):725-733.

13. Schwendimann R, Milisen K, Bühler H, De Geest S. Fall prevention in a Swiss acute care hospital setting Reducing multiple falls. $J$ Gerontol Nurs. 2006;32(3):13-22.

14. Williams TA, King G, Hill AM, et al. Evaluation of a falls prevention programme in an acute tertiary care hospital. J Clin Nurs. 2007;16(2):316-324.

15. Agency for Healthcare Research and Quality. Preventing Falls in Hospitals: A Toolkit for Improving Quality of Care. Rockville, MD: Agency for Healthcare Research and Quality; 2013.

16. Currie L. Chapter 10: Fall injury and prevention. In: Patient Safety and Quality: An Evidence-Based Handbook for Nurses. Rockville, MD: Agency for Healthcare Research and Quality; 2008:1-56. Available from: www.ahrq.gov/qual/nurseshdbk/docs/CurrieL_FIP.pdf. Accessed February 2, 2014.

17. National Health Service National Patient Safety Agency. Summary of the Third Report from the Patient Safety Observatory: Slips, Trips and Falls in Hospital. Leeds, UK: National Health Service; 2007. Available from: http://www.nrls.npsa.nhs.uk/resources/?EntryId45=59821. Accessed March 8, 2014.

18. Schwendimann R, Bühler H, De Geest S, Milisen K. Falls and consequent injuries in hospitalized patients: effects of an interdisciplinary falls prevention program. BMC Health Serv Res. 2006;6:69.

19. Tzeng HM, Yin CY. Exploring post-fall audit report data in an acute care setting. Clin Nurs Res. 2014. In press.

20. Butcher L. The no-fall zone. Hosp Health Netw. 2013;87(6):26-30, 31.

21. Cameron ID, Gillespie LD, Robertson MC, et al. Interventions for preventing falls in older people in care facilities and hospitals. Cochrane Database Syst Rev. 2012;12:CD005465.

22. Miake-Lye IM, Hempel S, Ganz DA, Shekelle PG. Inpatient fall prevention programs as a patient safety strategy: a systematic review. Ann Intern Med. 2013;158(5 Pt 2):390-396.

23. Raeder K, Siegmund U, Grittner U, Dassen T, Heinze C. The use of fall prevention guidelines in German hospitals - a multilevel analysis. J Eval Clin Pract. 2010;16(3):464-469.

24. Spoelstra SL, Given BA, Given CW. Fall prevention in hospitals: an integrative review. Clin Nurs Res. 2012;21(1):92-112.

25. Weinberg J, Proske D, Szerszen A, et al. An inpatient fall prevention initiative in a tertiary care hospital. Jt Comm J Qual Patient Saf. 2011; 37(7):317-325.

26. Charmel PA, Frampton SB. Building the business case for patientcentered care. Healthc Financ Manage. 2008;62(3):80-85.

27. Coulter A, Ellins J. Effectiveness of strategies for informing, educating, and involving patients. BMJ. 2007;335(7609):24-27.

28. Maurer M, Dardess P, Carman KL, Smeeding L. Guide to Patient and Family Engagement: Environmental Scan Report. Rockville, MD: Agency for Healthcare Research and Quality; 2012. Available from: http://www.ahrq.gov/research/findings/final-reports/ptfamilyscan/ ptfamilyscan.pdf. Accessed February 2, 2014. 
29. Greysen SR, Khanna RR, Jacolbia R, Lee HM, Auerbach AD. Tablet computers for hospitalized patients: A pilot study to improve inpatient engagement. J Hosp Med. Epub 2014 Feb 13.

30. Caligtan CA, Carroll DL, Hurley AC, Gersh-Zaremski R, Dykes PC. Bedside information technology to support patient-centered care. Int J Med Inform. 2012;81(7):442-451.

31. Dykes PC, Carroll DL, Hurley AC, et al. Building and testing a patient-centric electronic bedside communication center. J Gerontol Nurs. 2013;39(1):15-19.

32. Vawdrey DK, Wilcox LG, Collins SA, et al. A tablet computer application for patients to participate in their hospital care. AMIA Annu Symp Proc. 2011;2011:1428-1435.

33. Haines TP, Hill AM, Hill KD, et al. Patient education to prevent falls among older hospital inpatients: a randomized controlled trial. Arch Intern Med. 2011;171(6):516-524.

34. Jarousse LA [webpage on the Internet]. Executive dialogue: accelerating patient-centered care. Chicago, IL: American Hospital Association, Hospitals and Health Networks; 2013. Available from: http:/www.hhnmag.com/hhnmag/jsp/articledisplay.jsp?dcrpath=HHNMAG/Article/ data/07JUL2013/0713HHN_FEA_ExeDia\&domain=HHNMAG. Accessed February 2, 2014.

35. Centers for Disease Control and Prevention. Summary Health Statistics for the U.S. Population: National Health Interview Survey, 2011. Hyattsville, MD: U.S. Department of Health and Human Services; 2012. Available from: http://www.cdc.gov/nchs/data/series/sr_10/sr10_255. pdf. Accessed February 2, 2014.
36. Hendrich AL, Bender PS, Nyhuis A. Validation of the Hendrich II Fall Risk Model: a large concurrent case/control study of hospitalized patients. Appl Nurs Res. 2003;16(1):9-21.

37. Learn Not To Fall [webpage on the Internet]. Review your risk now. Learn Not To Fall; 2012. Available from: http://www.learnnottofall. com/. Accessed February 2, 2014.

38. Minnesota Hospital Association. Preventing Falls in the Hospital. Saint Paul, MN: Minnesota Hospital Association; Available from: http:// www.mnhospitals.org/Portals/0/Documents/ptsafety/falls/English_ Patient_Education_Brochure.pdf. Accessed February 2, 2014.

39. Morse JM, Morse RM, Tylko SJ. Development of a scale to identify the fall-prone patient. Can J Aging. 1989;8(4):366-377.

40. Oliver D, Britton M, Seed P, Martin FC, Hopper AH. Development and evaluation of evidence based risk assessment tool (STRATIFY) to predict which elderly inpatients will fall: case-control and cohort studies. BMJ. 1997;315(7115):1049-1053.

41. Rubenstein LZ, Vivrette R, Harker JO, Stevens JA, Kramer BJ. Validating an evidence-based, self-rated fall risk questionnaire (FRQ) for older adults. J Safety Res. 2011;42(6):493-499.

42. Spokane Regional Health District [webpage on the Internet]. Fall free Spokane. Spokane, WA: Spokane Regional Health District; 2014. Available from: http://www.srhd.org/topics/falls.asp. Accessed February 2, 2014.

43. Vivrette RL, Rubenstein LZ, Martin JL, Josephson KR, Kramer BJ. Development of a fall-risk self-assessment for community-dwelling seniors. J Aging Phys Act. 2011;19(1):16-29.
Patient Preference and Adherence

\section{Publish your work in this journal}

Patient Preference and Adherence is an international, peer-reviewed, open access journal that focusing on the growing importance of patient preference and adherence throughout the therapeutic continuum. Patient satisfaction, acceptability, quality of life, compliance, persistence and their role in developing new therapeutic modalities and compounds to optimize

\section{Dovepress}

clinical outcomes for existing disease states are major areas of interest for the journal. This journal has been accepted for indexing on PubMed Central. The manuscript management system is completely online and includes a very quick and fair peer-review system, which is all easy to use. Visit http://www. dovepress.com/testimonials.php to read real quotes from published authors. 